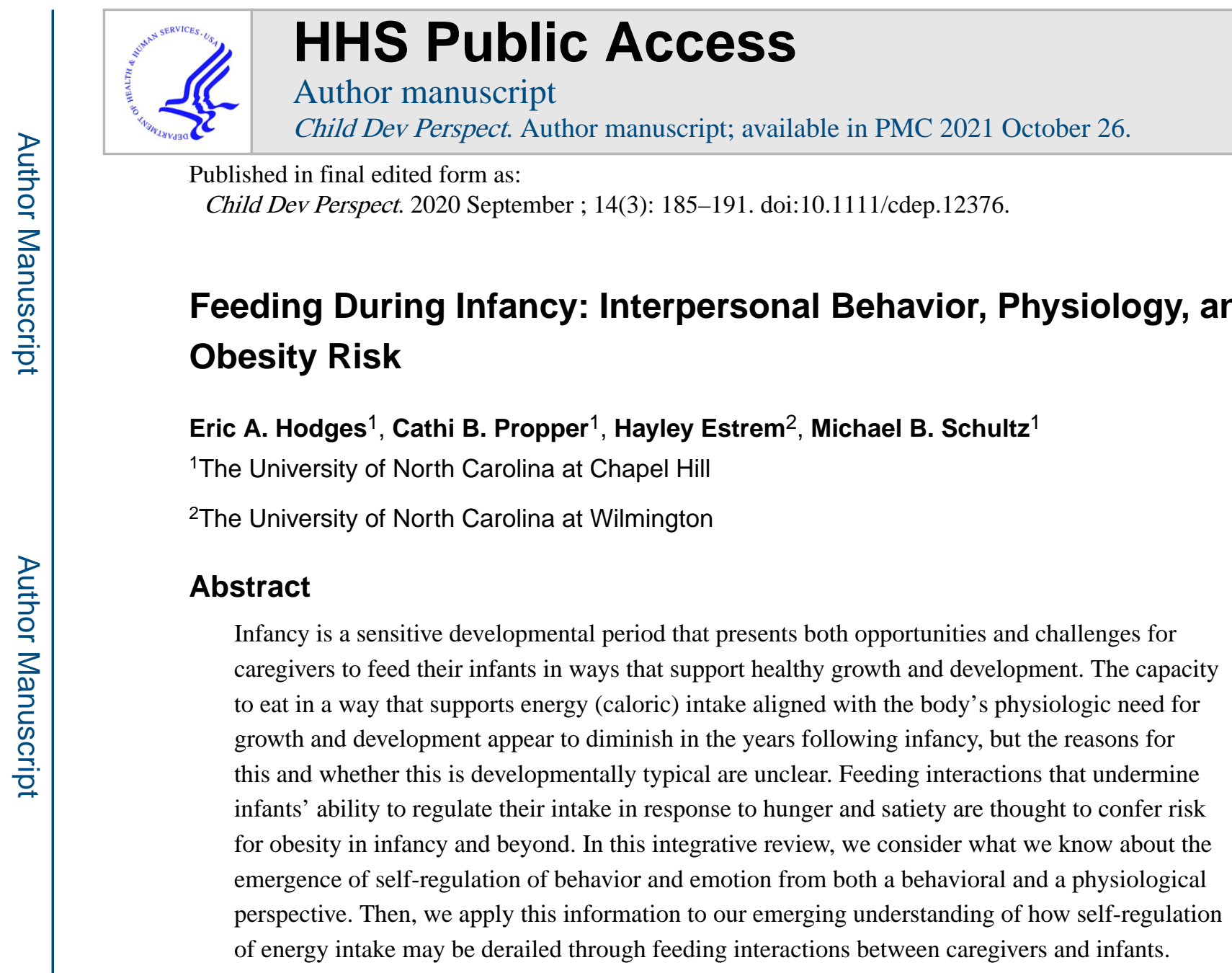

\title{
Keywords
}

parenting; infant; obesity

\begin{abstract}
Due to infants' initial dependence on caregivers and frequent need to eat throughout the day, infancy presents many opportunities and challenges to caregivers ${ }^{1}$ to support healthy growth and development. Feeding during this developmental stage presents a critical context that shapes eventual independent eating behavior and self-regulation of energy intake. Typically developing infants appear to have the capacity to adjust energy intake aligned with their body's physiologic need for growth and development (Fomon, Filmer, Thomas, Anderson, \& Nelson, 1975; Pearcey \& De Castro, 1997; Timby, Domellöf, Hernell, Lönnerdal, \& Domellöf, 2014), yet toddlers and older children demonstrate less self-regulation of energy intake (Cecil et al., 2005; Fox, Devaney, Reidy, Razafindrakoto, \& Ziegler, 2006; Kral et al., 2007; Smethers et al., 2019). Whether this is a typical developmental change is
\end{abstract}

\footnotetext{
Correspondence concerning this article should be addressed to Eric A. Hodges, Associate Professor, Robert Wood Johnson Foundation Nurse Faculty Scholar Alumnus, UNC-Chapel Hill School of Nursing, 5016 Carrington Hall, CB\# 7460, Chapel Hill, NC 27599-7460; eric.a.hodges@unc.edu.

Eric A. Hodges, School of Nursing, The University of North Carolina at Chapel Hill; Cathi B. Propper, Frank Porter Graham Child Development Institute, The University of North Carolina at Chapel Hill; Hayley Estrem, School of Nursing, The University of North Carolina at Wilmington; Michael B. Schultz, School of Nursing, The University of North Carolina at Chapel Hill.

${ }^{1}$ We refer to parents only when we mean this specifically and otherwise use the term caregivers to be inclusive of other family members who may serve as primary caregivers other than parents (e.g., grandparents).
} 
unclear. Chronically feeding infants in the absence of hunger or beyond satiety are thought to increase the risk of obesity during infancy, which may escalate the risk of subsequent obesity in childhood and adulthood (DiSantis, Hodges, Johnson, \& Fisher, 2011).

Obesity in early childhood affects a significant number of children and is associated with serious and potentially lifelong comorbidities, including prediabetes, type 2 diabetes mellitus, dyslipidemia, cardiovascular disease, anxiety, and depression (Kumar \& Kelly, 2017). Both becoming obese and gaining weight rapidly during infancy increase the likelihood that an infant will be obese later in life (Baird et al., 2005). The odds ratios or relative risks for later obesity if an infant is obese or gains weight rapidly are 1.359.38 and 1.17-5.70, respectively (Baird et al., 2005). Self-regulation of energy intake is shaped through feeding interactions during infancy and early childhood, with both shortterm implications (related to growth and adiposity) and potentially long-term (epigenetic) implications (Dalle Molle et al., 2017). The first 2 years of life may be critical for the development of self-regulation of energy intake (Kral et al., 2007), and are therefore an important target for preventing obesity (Birch, Parker, \& Burns, 2011).

Increasingly, research has been directed toward understanding the role of responsive feeding (i.e., feeding that responds to infants' signals of hunger and satiety cues; DiSantis et al., 2011) in improving feeding interactions during infancy and lowering the risk of obesity. While much of the focus of these studies has been at the level of parent-infant dyadic feeding behavior, less attention has been given to how such interactions may affect the nervous system's role in self-regulating energy intake (Sinopoulou, Harrold, \& Halford, 2016). While we acknowledge the complex physiologic processes that influence and are influenced by eating behavior, our interest is in the vagus nerve because of its role in regulating energy intake (de Lartigue \& $\mathrm{Xu}, 2018$ ) and its well-established associations with other aspects of behavioral self-regulation in infants (Porges \& Furman, 2011).

Therefore, in this integrative review, our goal is to synthesize findings from research on nutrition, physiology, and psychology to delineate factors that play a role in shaping self-regulation of energy intake and early risk for obesity stemming from parent-child interactions during infancy. First, we review what is known about the self-regulation of behavior and emotion, from both a behavioral and a physiological perspective, and apply this information to our emerging understanding of how self-regulation of energy intake develops during infancy. Then, we consider coregulatory processes within parent-infant dyads, the plasticity of the vagus nerve in early life, and how these may jointly influence self-regulation of energy intake and associated risk for obesity and adiposity. We conclude by identifying questions for the developmental science and behavioral nutrition community to consider.

\section{THE AUTONOMIC NERVOUS SYSTEM AND SELF-REGULATION}

The parasympathetic nervous system plays an important role in self-regulating various aspects of emotion, cognition, and behavior (Porges \& Furman, 2011). Cardiac vagal tonethe activity of the vagus nerve-is a key component of the paraysympathetic nervous system and therefore has been associated with these various domains of regulation. Heart rate 
variability (HRV) — beat-to-beat variation in heart rate—is a measurable index of cardiac vagal tone (Berntson et al., 1997). This variation is also synchronous with respiration (i.e., heart rate increases during inspiration and decreases during expiration) and is referred to as respiratory sinus arrhythmia (RSA). Although HRV and RSA are not the same, these terms are often used interchangeably to represent the contribution of the parasympathetic nervous system to self-regulation. To be consistent and clear, we use the term RSA to refer to findings related to parasympathetic influence on regulation.

Baseline RSA is a measure of resting autonomic state in the absence of environmental challenge and is considered a stable neurophysiological mechanism underlying autonomic and behavioral reactivity (Porges, Doussard-Roosevelt, Portales, \& Greenspan, 1996).

During restful periods, the vagus has an inhibitory influence on the heart, acting like a brake that increases vagal output to the sinoatrial (SA) node of the heart and reduces sympathetic influences, leading to a slower heart rate. Higher resting vagal tone during infancy has been associated with less temperamental difficulty, more sociable and explorative behavior, and greater self-regulation (see review by Field \& Diego, 2008). During times of environmental stress, these internal processes are disrupted and the brake on cardiac output is withdrawn, resulting in a decrease in vagal output to the SA node of the heart and an increase in heart rate. When environmental demands have ceased, the brake is re-engaged to promote decreases in metabolic output and a return to a calm state (Porges et al., 1996). The ability to effectively reduce RSA during a challenge (RSA withdrawal), followed by a return to baseline levels during calm and neutral times, may be an adaptive process. This may allow participation in demanding tasks by shifting attention from internal homeostatic demands to challenging external ones, supporting the use of coping strategies to control emotions or behavioral arousal (Porges et al., 1996). Withdrawal of RSA (larger decreases in RSA) during a challenging situation has been related to more successful emotion regulation in infants (Calkins, 1997; Porges et al., 1996) and caregivers (Moore et al., 2009).

\section{THE ROLE OF THE VAGUS NERVE IN HUNGER, SATIETY, EATING BEHAVIOR, AND OBESITY}

The vagus nerve also plays an important part in the self-regulation of energy intake, with its fundamental role being communication between the gut and brain in regard to hunger, satiety, and eating behavior (de Lartigue \& Xu, 2018; Kentish \& Page, 2014). This gut-brain communication involves the interplay of mechanoreceptors (responding to dis-tension and contraction of the stomach and small intestine), chemoreceptors (e.g., sensitive to intragastric acid levels), nutrient sensing (e.g., fat, protein, and carbohydrates), gastrointestinal peptides (e.g., ghrelin, which drives hunger and food intake, and leptin, which drives satiation), microbiota, and circadian rhythms (Kentish \& Page, 2014). The dynamic communication of these factors provides plasticity to vagal afferent neurons, which facilitates adaptive responses to internal and external stimuli around energy intake (de Lartigue \& Xu, 2018). Research on animals has revealed that dietary intake patterns that are rich in calories or high in fat can disrupt vagal afferent signaling, leading to weakened satiety signals relative to signals driving hunger and energy intake (Browning, Verheijden, \& Boeckxstaens, 2017; de Lartigue \& Xu, 2018); whether similar processes occur in humans 
remains to be seen. Nonetheless, taken together, these findings highlight the complex role the vagus nerve may play in human feeding and eating behavior that contributes to the induction and maintenance of obesity.

In adults, low baseline RSA (Coles, Vögele, Hilbert, \& Tuschen-Caffier, 2005) and less RSA withdrawal during a stressor (Friederich et al., 2006) have been associated with obesity. Thus, increased parasympathetic control in adult dieters may be a physiological marker of successful self-regulation of food intake (Meule, Lutz, Vögele, \& Kübler, 2012). Indeed, in one study, dieting success (i.e., self-control of food intake) was associated with increased parasympathetic control, even after controlling for body mass index, smoking, alcohol intake, and exercise (Meule et al., 2012). Similar patterns have been found among children: Decreased baseline RSA in 5- and 6-year olds (a purported indicator of less regulation) was associated with increased central adiposity (Vrijkotte et al., 2015). Similarly, children ( $\sim 5^{1 / 2}$ years mean age) who demonstrated lower levels of RSA withdrawal (a purported indicator of less regulation) during lab stressors were significantly more likely to be overweight 5 years later, though this finding held only among non-Hispanic Black children (Graziano, Calkins, Keane, \& O'Brien, 2011). Although reasons for the limitation of this phenomenon to non-Hispanic Black children were unclear, investigators underscored the importance of research into the relationship between children's eating behavior and cardiac regulation (Graziano et al., 2011).

\section{BEHAVIORAL AND PHYSIOLOGICAL REGULATION WITHIN THE PARENT- INFANT DYAD}

For infants, the caregiving environment is the most salient context for development and learning, and parental support can have longstanding consequences for developing selfregulation of behavior and emotion. The early emergence and development of self-regulation occurs within the context of caregiver-child interactions, during which dyadic experiences may support or derail behavioral self-regulation (Porges \& Furman, 2011; Porges et al., 1996). In fact, the quality of interactions during infancy affects the developing selfregulatory system so strongly that it "gets under the skin"; more responsive interactions have been associated with more effective regulation of the parasympathetic nervous system (Propper \& Holochwost, 2013).

In addition, the emotional quality of the early caregiving environment may influence the development of infant physiology (including RSA), especially during the early months of life when systems are developing and consolidating rapidly (Quigley \& Moore, 2018). For example, sensitive and responsive parenting has been associated with higher baseline RSA in infants, as well as more effective RSA withdrawal during a challenge, both of which have been associated with improved social-emotional, cognitive, and health outcomes (Bosquet Enlow et al., 2014; Moore et al., 2009). However, caregivers' behavior does not uni-laterally shape infants' developing physiology and behavior, but the affective and behavioral synchrony between infants and their caregivers may be most important (Feldman, Magori-Cohen, Galili, Singer, \& Louzoun, 2011; Moore \& Calkins, 2004). In one study, during moments of vocal and affect synchrony, the synchrony between mothers' and 
infants' heart rate patterns also increased (Feldman et al., 2011), suggesting that dyads coordinate their physiology and behavior through matching signals. In another study, infants' sympathetic and parasympathetic nervous systems covaried with those of their mothers upon reunion after mothers had been exposed to either a relaxing condition to promote parasympathetic reactivity or high stress to elicit sympathetic reactivity (Waters, West, Karnilowicz, \& Mendes, 2017).

Parent-child interactions are also crucial for developing feeding behaviors during infancy, which may lead to either well-regulated or dysregulated long-term behavior patterns (i.e., responsiveness to cues of hunger and satiety) that may have consequences for obesity risk. Infants develop and learn through inputs from experience-expectant and experiencedependent processes (Galván, 2010) that influence brain growth via structural and functional changes of neuronal circuits (neuronal plasticity). Experience-expectant processes are those experiences that are expected in typical development, such as exposure to language or visual stimulation, while experience-dependent processes are unique and specific to the individual, such as cultural activities or playing an instrument. Although feeding is an experience that occurs for all infants (experience-expectant), the specific experiences of feeding and the interactions with caregivers during feeding differ for all families and infants (experiencedependent). These feeding experiences occur not only every day, but many times a day, so they provide many opportunities for learning as the experiences accumulate. We propose that because of the plasticity of the brain and the vagus, and the rapid rate of myelination that happens in the first year of life (Porges \& Furman, 2011), repeated moment-to-moment parental responses to infants' feeding cues may act as experience-dependent processes that lead to neural changes and long-lasting effects on developing the perception of hunger and satiety.

\section{DYADIC INTERACTION, FEEDING, AND RISK OF OBESITY}

Beginning in infancy, interactions with caregivers shape both behavioral and physiological underpinnings of self-regulation, but we know little about how these interactions influence self-regulation of feeding, eating, and energy intake. A handful of studies suggest an association between RSA and the quality of the feeding interaction and type of feeding during infancy. In one study, healthy infants of mothers who were more sensitive to their infants' feeding cues and demonstrated warmth during feeding also demonstrated a more physiological adaptive response (using a measure of HRV) during feeding (Harrison, 2009). In a longitudinal study, during the Face-to-Face Still Face protocol (Tronick, Als, Adamson, Wise, \& Brazelton, 1978), a lab task designed to disrupt mother-infant interaction that often leads to distress in infants, breastfed infants demonstrated more RSA withdrawal than non-breastfed infants and breastfeeding mothers demonstrated higher baseline RSA than non-breastfeeding mothers (Quigley, Moore, Propper, Goldman, \& Cox, 2017). Finally, in another longitudinal study, breastfeeding infants demonstrated more stability in vagal tone (RSA) beginning at an earlier age than bottle-fed infants (Pivik et al., 2015). Although the authors of that study suggest that this difference may be due to the sensory and behavioral experiences that occur during breastfeeding versus bottle feeding, this interpretation should be regarded with caution given that the formula-fed infants also had shorter gestations, lower birth weights, and mothers with lower IQs who reported greater stress in their marriages. 
Nevertheless, though limited, these studies suggest the role that parent-infant interactions in the context of feeding may play in vagal regulation. This is an integral component of our theoretical orientation, which is guided by a model linking feeding responsiveness to obesity (see Figure 1).

This model is situated within the larger relational developmental systems paradigm, in which the biological, psychological, and sociocultural systems interpenetrate and mutually influence one another over the course of development (Overton, 2012). This paradigm requires us to acknowledge that energy intake self-regulation is modifiable by relational developmental processes. In our dyadic model, in addition to caregivers' responsiveness to cues, infants are responsible for the clarity of cues. Factors that may affect infants' cues and caregivers' perceptions of them include infants' temperament (Stifter \& Moding, 2018) and heritable appetite traits, such as perceived enjoyment of eating, demandingness during feeding, responsiveness to fullness, and pace of eating (Llewellyn, van Jaarsveld, Johnson, Carnell, \& Wardle, 2010).

A growing body of research suggests that infants' interpretation of internal states of hunger and satiety develops through social feeding interactions (Fotopoulou \& Tsakiris, 2017; Harshaw, 2008). Infants learn that the visceral sensations felt in hunger are alleviated through eating, which is controlled by their caregivers. They also have the opportunity to pair feelings of fullness with cessation of eating. The perception of internal states, such as hunger and fullness, is known as interoception (Stevenson, Mahmut, \& Rooney, 2015). Discordant feeding responsiveness, characterized by a chronic mismatch between a caregiver's feeding behavior and the infant's state (e.g., feeding in the absence of hunger or feeding beyond fullness), is thought to contribute to obesity by undermining the infant's ability to self-regulate intake (DiSantis et al., 2011). Indeed, in an analysis of parental responses evoked by infants, parents' use of food to soothe their infants has been associated with weight gain in infancy (Jansen et al., 2019; Stifter \& Moding, 2018).

We propose that feeding in the absence of hunger or beyond fullness may undermine self-regulation of energy intake not only through learning about eating that takes place in dyadic feeding interactions but also in the function of the vagus nerve in communicating hunger and satiety to the brain. Because infants are initially dependent, caregivers control what is offered to eat in addition to other factors, such as timing and duration of feeding. Such control most certainly interacts with the mechanoreceptors, chemoreceptors, nutrient sensing, gastrointestinal peptides, microbiota, and circadian rhythms of the vagus.

\section{DISCUSSION}

In this review, we sought to synthesize findings from physiologic, psychologic, and behavioral nutrition research to delineate the role that the interactive process of feeding during infancy may play in developmental risk for obesity. In light of the literature we reviewed, intraindividual vagally mediated physiologic processes that drive ingestive behavior appear to be modifiable within the context of relational dynamics between parents and infants. Infants bring biologically based characteristics, including temperament (Stifter \& Moding, 2018) and appetitive traits (Llewellyn et al., 2010), to the relationship, and 
are dependent on the feeder not only regarding what food is offered, but in terms of the frequency of offering, duration, amount, method, and caregivers' responsiveness to infants' feeding cues (DiSantis et al., 2011).

For most infants, innate drives of hunger are accompanied by feelings of satiety when this need is satisfied, yet a tremendous amount of learning occurs during infancy about what these sensations are and how alleviation of hunger and achievement of satiation occurs, and that learning depends on context (Harshaw, 2008). Since feeding occurs several times daily during infancy, feeding interactions present opportunities for infants to learn about hunger and satiety. As a result of the plasticity of the vagus in regard to ingestive behavior and the rapid rate of myelination of the vagus that occurs during the first 6 months (Porges \& Furman, 2011), it is quite conceivable that discordant feeding responsiveness (feeding in the absence of hunger or feeding beyond fullness) during infancy could lead to developmental disturbances in perception of hunger and satiety. This could, in turn, increase the risk of subsequent obesity during the early years of life as infants develop from complete dependence to increasing independence in eating.

This review deepens our understanding of the interplay of behavior and underlying autonomic physiology that supports communication of hunger and satiety to the brain. We have provided a window into the whole person that looks not only at behavior and interoception, but also at the role the vagus nerve may play and how it might also be modified in ways that increase the development and maintenance of the risk of obesity.

Our review has limitations. Although we focused primarily on the influence of the quality of the caregiver-child interaction on the vagus as a predictor of self-regulation of energy intake and risk for obesity and as a potential marker of the quality of parent-child interaction, we recognize that the empirical evidence supporting these links is sparse. In addition, other potential influences on ingestive behavior, such as epigenetic changes, are promising and intriguing, but beyond the scope of this review.

\section{LOOKING AHEAD}

Our review raises questions for the developmental science and behavioral nutrition community to consider. Bidirectionality in interpersonal physiology has implications. While much research on responsive feeding has focused on caregivers' effects on infants, a dyadic model suggests that infants evoke behavioral and physiologic responses in their caregivers and respond to caregivers' behavior and physiology, too. Researchers need to develop methods to assess dyadic behavior and physiology synchronously during feeding. Such research would facilitate investigation into the effects of feeding dynamics on caregivers' behavior, health, dyadic health, and family health. We also need to consider larger systems in which infants and their parents are embedded and take into account the roles social determinants of health and policies may play in shaping feeding and obesity risk.

Another area of research involves identifying characteristics of infants and dyads with differing trajectories in a context of discordant feeding responsiveness. Not all infants who experience suboptimal feeding develop obesity; some actually exhibit failure to thrive, in 
which they fail to gain adequate weight or lose a significant amount of weight over a sustained period (Ammaniti, Ambruzzi, Lucarelli, Cimino, \& D'Olimpio, 2004; Benoit, 2000), while others do not develop either condition. Because we cannot predict who will develop obesity or failure to thrive, retrospective analyses of groups with differing growth trajectories would be a good place to start.

Finally, measuring hunger and satiety signaling in the gut-brain axis during infancy presents a number of challenges. While neuroimaging and biomarkers such as blood glucose, leptin, and ghrelin have been used to assess satiation in older people (de Graaf, Blom, Smeets, Stafleu, \& Hendriks, 2004), using such markers with infants presents feasibility challenges. Our review suggests that measuring vagal tone may help because it is non-invasive, but the specificity with which this measure captures the hunger and satiety communication functions of the vagus remains to be determined.

Self-regulation is an important predictor of early feeding behavior and risk for obesity, yet researchers debate whether self-regulation is domain general, encompassing many forms of regulation, or made up of various subdomains (Nigg, 2017). Our integrative review and theoretical model are situated within contexts of feeding, nutrition, and regulation of energy intake. Whether the information and model we have presented have applications in domain-general self-regulation remains an empirical question and an important direction for the field. The answer will aid development of appropriate targets for obesity prevention and intervention once we know more about where energy intake regulation falls on the domain-general and domain-specific spectrum.

\section{CONCLUSION}

Consideration of levels of physiologic and interactive organization in infants and between infants and caregivers highlights the role that feeding behavior may have in shaping subsequent development of preventable disease. As a discipline, developmental science is interested not only in understanding mechanisms that lead to particular outcomes but also in applying such knowledge to optimize trajectories and improve health. Our review of behavior, physiology, and obesity risk contributes to these efforts by synthesizing what we know and suggesting areas of ongoing research to optimize health.

\section{Acknowledgments}

Dr. Eric A. Hodges' and Dr. Cathi B. Propper's work on this article was supported by the Eunice Kennedy Shriver National Institute of Child Health and Human Development (R21HD082707).

\section{REFERENCES}

Ammaniti M, Ambruzzi AM, Lucarelli L, Cimino S, \& D’Olimpio F (2004). Malnutrition and dysfunctional mother-child feeding interactions: Clinical assessment and research implications. Journal of the American College of Nutrition, 23, 259-271. 10.1080/07315724.2004.10719369 [PubMed: 15190051]

Baird J, Fisher D, Lucas P, Kleijnen J, Roberts H, \& Law C (2005). Being big or growing fast: Systematic review of size and growth in infancy and later obesity. British Medical Journal, 331, 929. 10.1136/bmj.38586.411273.E0 [PubMed: 16227306] 
Benoit D (2000).Feeding disorders, failure to thrive, and obesity (2nd ed.). In Zeanah CH Jr. (Ed.). New York, NY: Guilford.

Berntson GG, Bigger JT, Eckberg DL, Grossman P, Kaufmann PG, Malik M, ... van der Molen MW (1997). Heart rate variability: Origins, methods, and interpretive caveats. Psychophysiology, 34, 623-648. 10.1111/j.1469-8986.1997.tb02140.x [PubMed: 9401419]

Birch LL, Parker L, \& Burns A (Eds.). (2011). Early childhood obesity prevention policies. Washington, DC: National Academies Press. 10.17226/13124

Bosquet Enlow M, King L, Schreier HM, Howard JM, Rosenfield D, Ritz T, \& Wright RJ (2014). Maternal sensitivity and infant autonomic and endocrine stress responses. Early Human Development, 90, 377-385. 10.1016/j.earlhumdev.2014.04.007 [PubMed: 24794304]

Browning KN, Verheijden S, \& Boeckxstaens GE (2017). The vagus nerve in appetite regulation, mood, and intestinal inflammation. Gastroenterology, 152, 730-744. 10.1053/j.gastro.2016.10.046 [PubMed: 27988382]

Calkins SD (1997). Cardiac vagal tone indices of temperamental reactivity and behavioral regulation in young children. Developmental Psychobiology, 31, 125-135. 10.1002/ (SICI)1098-2302(199709)31:2<125:AID-DEV5>3.0.CO;2-M [PubMed: 9298638]

Cecil JE, Palmer CNA, Wrieden W, Murrie I, Bolton-Smith C, Watt P, ... Hetherington MM (2005). Energy intakes of children after preloads: Adjustment, not compensation. The American Journal of Clinical Nutrition, 82, 302-308. 10.1093/ajcn.82.2.302 [PubMed: 16087972]

Coles J, Vögele C, Hilbert A, \& Tuschen-Caffier B (2005). Fasten und (Über)-essen [Fasting and (over)-eating]. Zeitschrift furüKlinis che Psychologie und Psychotherapie [Journal of Clinical Psychology and Psychotherapy], 34, 95-103. 10.1026/1616-3443.34.2.95

Dalle Molle R, Fatemi H, Dagher A, Levitan RD, Silveira PP, \& Dubé L (2017). Gene and environment interaction: Is the differential susceptibility hypothesis relevant for obesity? Neuroscience and Biobehavioral Reviews, 73, 326-339. 10.1016/j.neubiorev.2016.12.028 [PubMed: 28024828]

de Graaf C, Blom WAM, Smeets PAM, Stafleu A, \& Hendriks HFJ (2004). Biomarkers of satiation and satiety. The American Journal of Clinical Nutrition, 79, 946-961. 10.1093/ajcn/79.6.946 [PubMed: 15159223]

de Lartigue G, \& Xu C (2018). Mechanisms of vagal plasticity influencing feeding behavior. Brain Research, 1693, 146-150. 10.1016/j.brainres.2018.03.030 [PubMed: 29903616]

DiSantis KI, Hodges EA, Johnson SL, \& Fisher JO (2011). The role of responsive feeding in overweight during infancy and toddlerhood: A systematic review. International Journal of Obesity, 35, 480-492. 10.1038/ijo.2011.3 [PubMed: 21427696]

Feldman R, Magori-Cohen R, Galili G, Singer M, \& Louzoun Y (2011). Mother and infant coordinate heart rhythms through episodes of interaction synchrony. Infant Behavior \& Development, 34, 569-577. 10.1016/j.infbeh.2011.06.008 [PubMed: 21767879]

Field T, \& Diego M (2008). Vagal activity, early growth and emotional development. Infant Behavior \& Development, 31, 361-373. 10.1016/j.infbeh.2007.12.008 [PubMed: 18295898]

Fomon SJ, Filmer IJ, Thomas LN, Anderson TA, \& Nelson SE (1975). Influence of formula concentration on caloric intake and growth of normal infants. Acta Paediatrica, 64, 172-181. 10.1111/j.1651-2227.1975.tb03818.x

Fotopoulou A, \& Tsakiris M (2017). Mentalizing homeostasis: The social origins of interoceptive inference. Neuropsychoanalysis, 19, 3-28. 10.1080/15294145.2017.1294031

Fox MK, Devaney B, Reidy K, Razafindrakoto C, \& Ziegler P (2006). Relationship between portion size and energy intake among infants and toddlers: Evidence of self-regulation. Journal of the American Dietetic Association, 106, S77-S83. 10.1016/j.jada.2005.09.039 [PubMed: 16376632]

Friederich HC, Schild S, Schellberg D, Quenter A, Bode C, Herzog W, \& Zipfel S (2006). Cardiac parasympathetic regulation in obese women with binge eating disorder. International Journal of Obesity, 30, 534-542. 10.1038/sj.ijo.0803181 [PubMed: 16314876]

Galván A (2010). Neural plasticity of development and learning. Human Brain Mapping, 31, 879-890. 10.1002/hbm.21029 [PubMed: 20496379] 
Graziano PA, Calkins SD, Keane SP, \& O’Brien M (2011). Cardiovascular regulation profile predicts developmental trajectory of BMI and pediatric obesity. Obesity, 19, 1818-1825. 10.1038/ oby.2011.98 [PubMed: 21546929]

Harrison TM (2009). Effect of maternal behavior on regulation during feeding in healthy infants and infants with transposition. Journal of Obstetric, Gynecologic \& Neonatal Nursing, 38, 504-513. 10.1111/j.1552-6909.2009.01045.x

Harshaw C (2008). Alimentary epigenetics: A developmental psychobiological systems view of the perception of hunger, thirst and satiety. Developmental Review, 28, 541-569. 10.1016/ j.dr.2008.08.001 [PubMed: 19956358]

Jansen PW, Derks IPM, Batenburg A, Jaddoe VWV, Franco OH, Verhulst FC, \& Tiemeier H (2019). Using food to soothe in infancy is prospectively associated with childhood BMI in a populationbased cohort. The Journal of Nutrition, 149, 788-794. 10.1093/jn/nxy277 [PubMed: 30989177]

Kentish SJ, \& Page AJ (2014). Plasticity of gastro-intestinal vagal afferent endings. Physiology \& Behavior, 136, 170-178. 10.1016/j.physbeh.2014.03.012 [PubMed: 24657740]

Kral TVE, Stunkard AJ, Berkowitz RI, Stallings VA, Brown DD, \& Faith MS (2007). Daily food intake in relation to dietary energy density in the free-living environment: A prospective analysis of children born at different risk of obesity. The American Journal of Clinical Nutrition, 86, 41-47. 10.1093/ajcn/86.1.41 [PubMed: 17616761]

Kumar S, \& Kelly AS (2017). Review of childhood obesity: From epidemiology, etiology, and comorbidities to clinical assessment and treatment. Mayo Clinic Proceedings, 92, 251-265. 10.1016/j.mayocp.2016.09.017 [PubMed: 28065514]

Llewellyn CH, van Jaarsveld CHM, Johnson L, Carnell S, \& Wardle J (2010). Nature and nurture in infant appetite: Analysis of the Gemini twin birth cohort. The American Journal of Clinical Nutrition, 91, 1172-1179. 10.3945/ajcn.2009.28868 [PubMed: 20335548]

Meule A, Lutz A, Vögele C, \& Kübler A (2012). Food cravings discriminate differentially between successful and unsuccessful dieters and non-dieters. Validation of the food cravings questionnaires in German. Appetite, 58, 88-97. 10.1016/j.appet.2011.09.010 [PubMed: 21983051]

Moore GA, \& Calkins SD (2004). Infants' vagal regulation in the still-face paradigm is related to dyadic coordination of mother-infant interaction. Developmental Psychology, 40, 1068. 10.1037/0012-1649.40.6.1068 [PubMed: 15535757]

Moore GA, Hill-Soderlund AL, Propper CB, Calkins SD, Mills-Koonce WR, \& Cox MJ (2009). Mother-infant vagal regulation in the face-to-face still-face paradigm is moderated by maternal sensitivity. Child Development, 80, 209-223. 10.1111/j.1467-8624.2008.01255.x [PubMed: 19236402]

Nigg JT (2017). Annual research review: On the relations among self-regulation, self-control, executive functioning, effortful control, cognitive control, impulsivity, risk-taking, and inhibition for developmental psychopathology. Journal of Child Psychology and Psychiatry, and Allied Disciplines, 58, 361-383. 10.1111/jcpp.12675

Overton WF (2012). Relationism and relational developmental systems: A paradigm for developmental science in the post-Cartesian era. Advances in Child Development and Behavior, 44, 21-64. 10.1016/B978-0-12-397947-6.00002-7

Pearcey SM, \& De Castro JM (1997). Food intake and meal patterns of one year old infants. Appetite, 29, 201-212. 10.1006/appe.1997.0099 [PubMed: 9344428]

Pivik RT, Andres A, Tennal KB, Gu Y, Cleves MA, \& Badger TM (2015). Infant diet, gender and the development of vagal tone stability during the first two years of life. International Journal of Psychophysiology, 96, 104-114. 10.1016/j.ijpsycho.2015.02.028 [PubMed: 25746938]

Porges SW, Doussard-Roosevelt JA, Portales AL, \& Greenspan SI (1996). Infant regulation of the vagal "brake" predicts child behavior problems: A psychobiological model of social behavior. Developmental Psychobiology, 29, 697-712. 10.1002/(SICI)1098-2302(199612)29:8<697:AIDDEV5>3.0.CO;2-O [PubMed: 8958482]

Porges SW, \& Furman SA (2011). The early development of the autonomic nervous system provides a neural platform for social behaviour: A polyvagal perspective. Infant and Child Development, 20, 106-118. 10.1002/icd.688 [PubMed: 21516219] 
Propper CB, \& Holochwost SJ (2013). The influence of proximal risk on the early development of the autonomic nervous system. Developmental Review, 33, 151-167. 10.1016/j.dr.2013.05.001

Quigley KM, \& Moore GA (2018). Development of cardiac autonomic balance in infancy and early childhood: A possible pathway to mental and physical health outcomes. Developmental Review, 49, 41-61. 10.1016/j.dr.2018.06.004

Quigley KM, Moore GA, Propper CB, Goldman BD, \& Cox MJ (2017). Vagal regulation in breastfeeding infants and their mothers. Child Development, 88, 919-933. 10.1111/cdev.12641 [PubMed: 27800619]

Sinopoulou V, Harrold J, \& Halford J (2016). Meaning and assessment of satiety in childhood. In The ECOG's ebook on child and adolescent obesity (pp. 1-17). European Childhood Obesity Group. Retrieved from http://ebook.ecog-obesity.eu/wp-content/uploads/2015/02/ECOG-ObesityeBook-Meaning-And-Assessment-Of-Satiety-In-Childhood.pdf

Smethers AD, Roe LS, Sanchez CE, Zuraikat FM, Keller KL, \& Rolls BJ (2019). Both increases and decreases in energy density lead to sustained changes in preschool children's energy intake over 5 days. Physiology \& Behavior, 204, 210-218. 10.1016/j.physbeh.2019.02.042 [PubMed: 30831180]

Stevenson RJ, Mahmut M, \& Rooney K (2015). Individual differences in the interoceptive states of hunger, fullness and thirst. Appetite, 95, 44-57. 10.1016/j.appet.2015.06.008 [PubMed: 26119812]

Stifter CA, \& Moding KJ (2018). Infant temperament and parent use of food to soothe predict change in weight-for-length across infancy: Early risk factors for childhood obesity. International Journal of Obesity, 42, 1631-1638. 10.1038/s41366-018-0006-4 [PubMed: 29463917]

Timby N, Domellöf E, Hernell O, Lönnerdal B, \& Domellöf M (2014). Neurodevelopment, nutrition, and growth until 12 mo of age in infants fed a low-energy, low-protein formula supplemented with bovine milk fat globule membranes: A randomized controlled trial. The American Journal of Clinical Nutrition, 99, 860-868. 10.3945/ajcn.113.064295 [PubMed: 24500150]

Tronick E, Als H, Adamson L, Wise S, \& Brazelton TB (1978). The infant's response to entrapment between contradictory messages in face-to-face interaction. Journal of the American Academy of Child Psychiatry, 17, 1-13. 10.1016/s0002-7138(09)62273-1 [PubMed: 632477]

Vrijkotte TGM, van den Born B-JH, Hoekstra CMCA, Gademan MGJ, van Eijsden M, de Rooij SR, \& Twickler MTB (2015). Cardiac autonomic nervous system activation and metabolic profile in young children: The ABCD study. PLoS One, 10, e0138302. 10.1371/journal.pone.0138302 [PubMed: 26394362]

Waters SF, West TV, Karnilowicz HR, \& Mendes WB (2017). Affect contagion between mothers and infants: Examining valence and touch. Journal of Experimental Psychology. General, 146, 1043-1051. 10.1037/xge0000322 [PubMed: 28493755] 


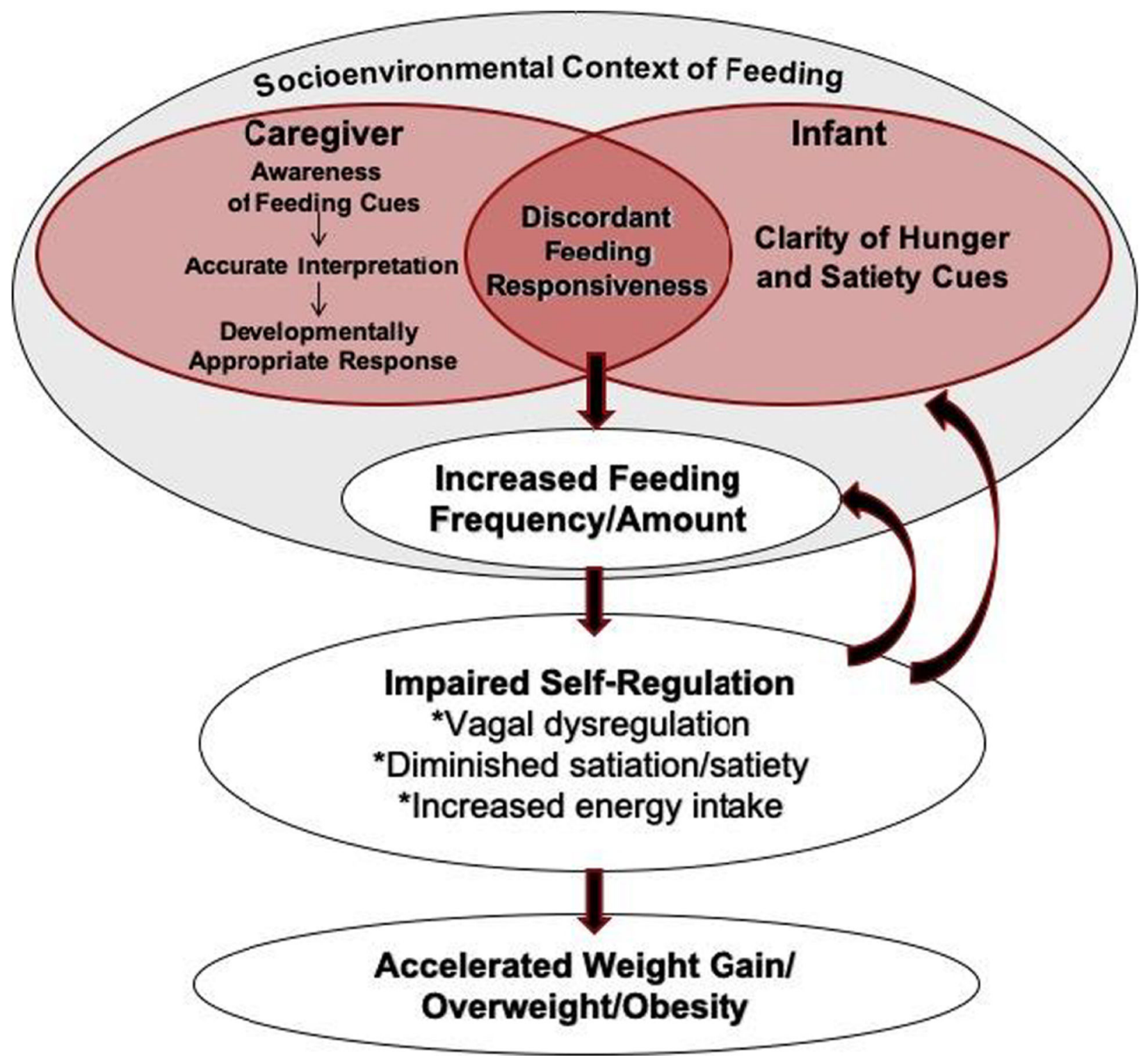

Figure 1.

Discordant feeding responsiveness and energy intake self-regulation. This figure illustrates the theoretical relationships among discordant feeding responsiveness; energy intake selfregulation; and accelerated weight gain, overweight, and obesity risk. Adapted from DiSantis et al. (2011). 